\title{
Minimum length scale in topology optimization by geometric constraints
}

\section{Zhou, Mingdong; Lazarov, Boyan Stefanov; Wang, Fengwen; Sigmund, Ole}

\section{Published in:}

Computer Methods in Applied Mechanics and Engineering

Link to article, DOI:

10.1016/j.cma.2015.05.003

Publication date:

2015

Document Version

Peer reviewed version

Link back to DTU Orbit

Citation (APA):

Zhou, M., Lazarov, B. S., Wang, F., \& Sigmund, O. (2015). Minimum length scale in topology optimization by geometric constraints. Computer Methods in Applied Mechanics and Engineering, 293, 266-282. https://doi.org/10.1016/j.cma.2015.05.003

\section{General rights}

Copyright and moral rights for the publications made accessible in the public portal are retained by the authors and/or other copyright owners and it is a condition of accessing publications that users recognise and abide by the legal requirements associated with these rights.

- Users may download and print one copy of any publication from the public portal for the purpose of private study or research.

- You may not further distribute the material or use it for any profit-making activity or commercial gain

- You may freely distribute the URL identifying the publication in the public portal 


\title{
Minimum length scale in topology optimization by geometric constraints
}

\author{
Mingdong Zhou*, Boyan S. Lazarov, Fengwen Wang, Ole Sigmund \\ Solid Mechanics, Department of Mechanical Engineering, Technical University of Denmark, Nils \\ Koppels Allé, Building 404, DK-2800 Kgs. Lyngby, Denmark
}

\begin{abstract}
A density-based topology optimization approach is proposed to design structures with strict minimum length scale. The idea is based on using a filtering-threshold topology optimization scheme and computationally cheap geometric constraints. The constraints are defined over the underlying structural geometry represented by the filtered and physical fields. Satisfying the constraints leads to a design that possesses user-specified minimum length scale. Conventional topology optimization problems can be augmented with the proposed constraints to achieve minimum length scale on the final design. No additional finite element analysis is required for the constrained optimization. Several benchmark examples are presented to show the effectiveness of this approach.
\end{abstract}

Keywords: Minimum length scale, Topology optimization, Geometric constraint

\section{Introduction}

The aim of this paper is to design two-phase (solid-void) structures that possess strict minimum length scale by using geometric constraints. The constraints are defined based on the structural geometry represented by a filtering-threshold topology optimization scheme. They are computationally cheap and can be easily implemented into conventional topology optimization problems. No additional finite element analysis is required to achieve the prescribed minimum length scale on the final design.

\footnotetext{
*Corresponding author

Email address: minzho@mek . dtu .dk (Mingdong Zhou)

Preliminary results of this work was submitted / published to the congress of WCSMO-11
} 
Topology optimization $[1,2,3]$ has been applied to design optimized structures for a wealth of physics problems and paving its way towards a large number of industrial applications $[4,5]$. It is desirable that a topologically optimized design can be fabricated reliably by a certain manufacturing process. To fulfil1 the requirement for manufacturing, one recent trend is to directly consider the manufacturing characteristics in the optimization process $[6,7,8]$, i.e. to achieve minimum length scale on the optimized design and thus ensure prototype manufacturability. In additive manufacturing [6], if this condition is not satisfied, holes or disconnected parts may appear in the prototype. Another example is the design of compliant mechanisms, for which achieving minimum length scale is crucial to avoid the tiny-hinges at structural joints [2].

Some previous approaches to impose minimum length scale in topology optimization are compared as follows. Within the density-based topology optimization [2], Poulsen proposed the so-called MOLE (MOnotonicity based minimum LEngth scale) method [9] to impose minimum length scale onto the topologically optimized results. In this method, a constraint functional based on local density variation is formulated, which guarantees that the minimum length scale of the final design is larger than the size of a circular "looking glass". Guest [10] suggested projection schemes to achieve minimum length scale by projecting the nodal density into the element space with a supporting radius $r_{\text {min }}$, which stands for the minimum feature size. However, this scheme does not resolve the "one-node hinge" problem [2] in designing topologically optimized compliant mechanism$\mathrm{s}$, as it imposes length scale only on one phase [12]. Besides, simple projection may result in grey scale for some design problems [11]. The robust formulation$\mathrm{s}[11,12,13]$, which take the eroded, dilated and (one or several) intermediate design realizations into account at the same time, impose length scale on the intermediate blueprint design only if the considered design realizations share the same topology. However, as pointed out in [12], the robust formulation does not necessarily guarantee a consistent topology for the realizations in different physical problems and the length scale can only be checked a posteriori. Another drawback of using a robust formulation is the high computational cost, that it basically requires several finite analysis in every design iteration. A perturbation based technique [14] proposed by Lazarov et. al is a computationally efficient solution for the stochastic formulation based topology optimization which accounts for geometric variations. However, due to the locality of the approximation, it cannot provide a clear length-scale control for compliant mechanism problems. Recently, a skeleton-based idea, which is similar to that in [17] with the level set method, is implemented using a density based method in [18]. The idea is 
to extract the medial-zone of a structure and to constrain the density value (or the signed-distance function in [17]) for all points inside the zone. The constraints can impose minimum and maximum length scale in the optimized design. In both papers $[17,18]$, the sensitivity regarding the change of the medial-zone is neglected in the sensitivity analysis. Possible shortcomings of this approach are discussed in detail in [20].

Within the level set based method [15], Chen et al. [16] use a quadratical energy functional as a penalty term in the objective function, which favors a thin elongated structural layout with length scale in final results. However, the energy functional requires complex geometric information and there is no explicit way to define the exact length scale. A rigorous mathematic approach for imposing minimum and maximum length scale in level set based topology optimization is proposed in $[19,20]$. Besides the above approaches, predefined engineering features with length scale can be designed and optimized using a CSG based level set approach as discussed in [21]. Readers who are interested in maximum length scale in topology optimization are referred to the articles [17, 18, 19, 20, 22].

In this paper, a filtering-threshold topology optimization scheme (also known as three-field scheme) [3], which utilizes a design field $\rho(0 \leq \rho \leq 1)$, a filtered design field $\tilde{\rho}$ and a projected (physical) field $\bar{\rho}$, is adopted as the basic computational framework. The idea is motivated by the fact that minimum length scale can be imposed over the blueprint design $\bar{\rho}_{\eta_{i}}$ (thresholded by $\eta_{i}$ ) implicitly by using a robust formulation $[11,12,13]$, if all the physical realizations $\bar{\rho}_{\eta}$ thresholded in a range $\eta \in\left(\eta_{d}, \eta_{e}\right)\left(0<\eta_{d}<\eta_{i}<\eta_{e}<1\right)$ share a consistent topology. To achieve the latter, one sufficient condition is given as follows:

$$
\begin{array}{ll}
\text { (i) } \quad \tilde{\rho}(\mathbf{x}) \geq \eta_{e}, \quad \forall \mathbf{x} \in \Omega_{1}=\left\{\mathbf{x} \mid \bar{\rho}_{\eta_{i}}(\mathbf{x})=1 \text { and } \nabla \tilde{\rho}=0\right\} \\
\text { (ii) } \quad \tilde{\rho}(\mathbf{x}) \leq \eta_{d}, \quad \forall \mathbf{x} \in \Omega_{2}=\left\{\mathbf{x} \mid \bar{\rho}_{\eta_{i}}(\mathbf{x})=0 \text { and } \nabla \tilde{\rho}=0\right\}
\end{array}
$$

where $\Omega_{1}$ represents the inflection region of the filtered field in the solid phase of the physical field and $\Omega_{2}$ captures that in the void. Fig. 1 illustrates this idea with a 1D example. The solid curve represents an initial filtered field, for which the physical fields thresholded at $\eta_{d}, \eta_{i}, \eta_{e}$ possess different topologies. Re-designing it into the dashed curve as shown in the figure to satisfies $(i)$ and (ii), a solid phase is ensured in $\Omega_{1}$ for all the physical realizations thresholded by $\eta<\eta_{e}$ and a void phase remains in $\Omega_{2}$ within $\eta>\eta_{d}$. As a result, all the physical realizations thresholded by $\eta \in\left(\eta_{d}, \eta_{e}\right)$ share a consistent topology ${ }^{1}$ and minimum length scale

\footnotetext{
${ }^{1}$ Theoretically, a consistent topology is guaranteed within the range $\left(\eta_{d}, \eta_{e}\right)$ by using an ideal
} 


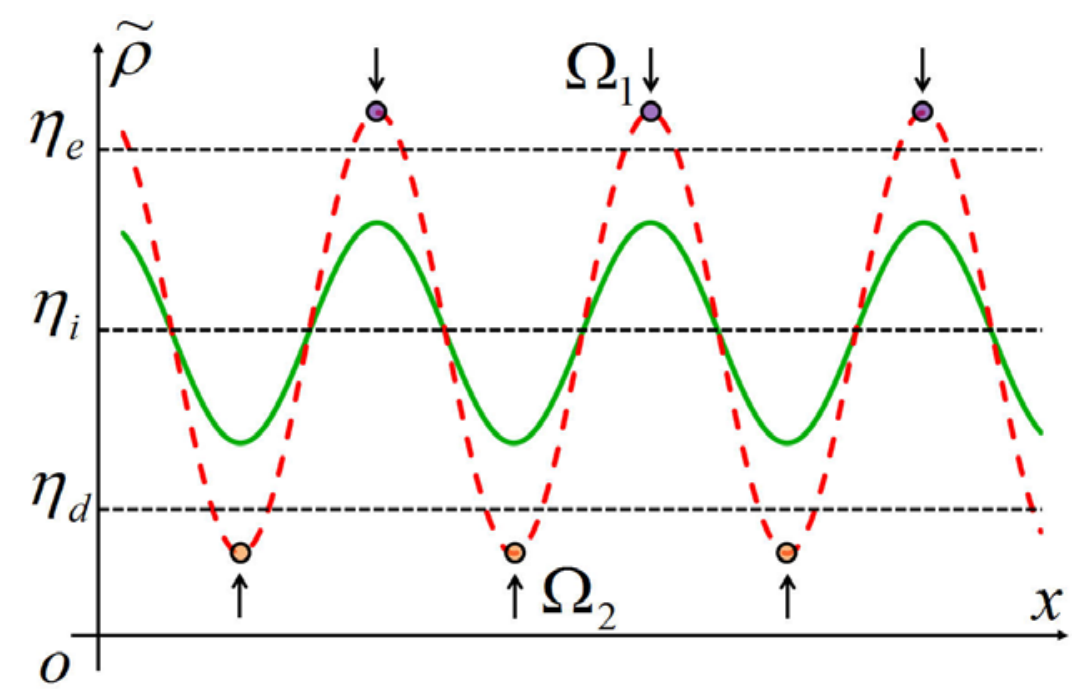

Figure 1: Re-designing a filtered field (from the solid to the dashed curve) to satisfy the conditions (i) - (ii) according to Eqs. (1) and (2). Minimum length scale is achieved on the physical field thresholded by $\eta_{i}$ for the dashed curve.

is expected on the blueprint design $\bar{\rho}_{\eta_{i}}$ for both solid and void phases. In this work, two geometric constraints are proposed to meet the above condition and minimum length scale is achieved by solving a constrained optimization problem. The approach is inspired by the works in $[17,18,19,20]$ but different from them. Partly only the minimum length-scale problem is considered here and partly the constraints here are formulated based on a filtering-threshold topology optimization scheme and they are differentiable w.r.t. the design variable. The overall scheme does not require additional finite element analysis comparing to the robust formulation $[11,12,13]$. It can be easily extended to existing density-based topology optimization frameworks. However, although the proposed scheme indeed ensures length scale, it does not guarantee robustness towards design variations, which is demonstrated with an example from nano-optics.

The remainder of this paper is organized as follows. In Section 2, the threefield topology optimization scheme and the geometric constraints are introduced.

Heaviside function. Due to the usage of a smoothed Heaviside function Eq. (7) in this paper, the physical realizations thresholded at $\eta \approx \eta_{e}$ and $\eta \approx \eta_{d}$ may contain grey elements with a small physical field value $\bar{\rho}<0.5$. This numerical issue does not jeopardize the effectiveness of this approach in imposing minimum length scale on the blueprint design. 
The formulation of the geometrically constrained optimization problem is presented afterwards. In Section 3, the proposed approach is demonstrated using several benchmark design problems, including a compliant mechanism, heat conduction and a slow light waveguide. Key issues are discussed and implementation details are provided. Conclusions are stated in Section 4.

\section{Topology optimization with geometric constraints}

\subsection{A three-field topology optimization formulation}

The goal of topology optimization is to find a material distribution in a given design domain that minimizes an objective function and satisfies constraints. The optimization problem generally reads in a discrete form [3] as:

$$
\begin{aligned}
\text { min. : } & F(\mathbf{u}(\boldsymbol{\rho}), \boldsymbol{\rho}), \\
\text { s.t. : } & g_{j}(\boldsymbol{\rho}) \leq 0, \quad j=1: m, \\
: & 0 \leq \boldsymbol{\rho} \leq 1,
\end{aligned}
$$

where $\rho$ denotes the design variable vector, $f$ is an objective functional, $\mathbf{u}$ is the state variable denoting the system response at the nodal points and $g$ is the constraint. For a linear elasticity problem, $\mathbf{u}$ is the solution of the state equation (as a constraint in the above formulation):

$$
\mathbf{K u}=\mathbf{f},
$$

where $\mathbf{K}$ and $\mathbf{f}$ are the structural stiffness matrix and the force vector, respectively. The Solid Isotropic Material interpolation with Penalization (SIMP) scheme [23] is adopted to model the element stiffness $\mathbf{K}_{i}$ for the element $i$ as:

$$
\mathbf{K}_{i}=\left(E_{\min }+\bar{\rho}_{i}^{p}\left(E_{0}-E_{\text {min }}\right)\right) \mathbf{K}_{0},
$$

where $\bar{\rho}_{i}$ is the value of the physical field (described below), $\mathbf{K}_{0}$ denotes the element stiffness matrix for unit stiffness, $E_{0}$ represents the Young's modulus of the material phase, $E_{\min }$ is a small number to avoid the numerical singularity issue in finite element analysis and $p$ is the penalization power. The SIMP method is widely applied to material properties interpolation in topology optimization for different physical problems [2].

The three-field topology optimization scheme utilizes a design field $\rho$, a filtered field $\tilde{\rho}$ and a physical field $\bar{\rho}$, whose relations are defined through the following filtering and threshold processes:

$$
\tilde{\rho}_{i}=\frac{\sum_{j \in \mathbb{N}_{i}} \omega\left(\mathbf{x}_{j}\right) v_{j} \rho_{j}}{\sum_{j \in \mathbb{N}_{i}} \omega\left(\mathbf{x}_{j}\right) v_{j}}, \quad \omega\left(\mathbf{x}_{j}\right)=R-\left|\mathbf{x}_{i}-\mathbf{x}_{j}\right|,
$$




$$
\bar{\rho}_{i}=\frac{\tanh (\beta \cdot \eta)+\tanh \left(\beta \cdot\left(\tilde{\rho}_{i}-\eta\right)\right)}{\tanh (\beta \cdot \eta)+\tanh (\beta \cdot(1.0-\eta))} .
$$

In Eq. (6), $\mathbb{N}_{i}$ is the neighborhood set of elements lying within the filter domain of the element $i, R$ is the radius of a linear hat-shape filter, $v_{j}$ is the volume of the element $j, \omega$ is a weighting function of the distance between the central coordinates $\mathbf{x}_{i}$ and $\mathbf{x}_{j}$ of the cell $i$ and $j$. In Eq. (7), $\beta$ controls the steepness of the approximated Heaviside function and $\eta$ is the threshold. Note that, other filters can serve for the same purpose as long as $\tilde{\rho}$ is smooth and the inflection regions $\Omega_{1}$ and $\Omega_{2}$ can be captured properly by the proposed structural indicators (c.f. Section 2.2).

\subsection{Structural indicator functions}

In order to identify the inflection regions $\Omega_{1}, \Omega_{2}$ defined in Eqs. (1-2), two structural indicator functions are proposed as follows:

$$
\begin{gathered}
I^{s}=\bar{\rho} \cdot \exp \left(-c \cdot|\nabla \tilde{\rho}|^{2}\right), \\
I^{v}=(1-\bar{\rho}) \cdot \exp \left(-c \cdot|\nabla \tilde{\rho}|^{2}\right),
\end{gathered}
$$

where the subscripts $s$ and $v$ stand for the solid and void phase, respectively. The exponential term in Eqs. (8) and (9) annotates the inflection region of a filtered field $(|\nabla \tilde{\rho}|=0)$ with value 1 , while the parameter $c$ controls the decay rate of $I^{s}$ and $I^{v}$ wherever $|\nabla \tilde{\rho}| \neq 0$.

Fig. 2 shows a cantilever beam example obtained by using the above filteringthreshold topology optimization scheme. A fixed Dirichlet boundary of $\mathbf{u}=0$ is imposed on the left side of the domain and a vertical point load is applied at the middle of the right side. Figs. 2(a-c) record the optimized design field $\rho$, filtered field $\tilde{\rho}(R=10 \times$ element size $)$ and physical field $\bar{\rho}(\beta=32, \eta=0.5)$, respectively. A uniform mesh of $400 \times 150$ quadrilateral elements ${ }^{2}$ is used in this example. The indicators $I^{s}$ and $I^{v}$ computed using different $c$ values are compared in Figs. 2(d-f) and (g-i) respectively. By using a large $c(=10000)$ as shown in Fig. 2(f,i), the proposed indicator function captures $\Omega_{1}$ and $\Omega_{2}$ more precisely than the others. When using a smaller $c$ (e.g. $c=200)$, the exponential term in Eqs. (8-9) decays slowly w.r.t. the gradient variation, such that the indicators as shown in Figs. 2(d,g) contain non-zero value in a "wider" area, including both the target inflection and some non-inflection regions.

With an ideal continuous-field representation, the parameter $c$ can be set infinitely large such that the indicators only capture the inflection regions with value

\footnotetext{
${ }^{2}$ Bilinear elements are used for all examples in this paper.
} 
1 but 0 otherwise. In practice, however, $\nabla \tilde{\rho}=0$ does not hold over the entire (discretized) inflection regions due to a finite discretization and numerically computing $\nabla \tilde{\rho}$ using finite difference. It is observed that the numerical error of $\nabla \tilde{\rho}$ at the inflection regions ranges approximately between $\nabla \tilde{\rho} \in[0,1.5] / r^{2}$ for $2 \mathrm{D}$ problems ${ }^{3}$, where $r=R / h$ and $h$ represents the element size. A too large $c$ value will fail in capturing those regions (e.g. $I^{s}, I^{v} \approx 0$ ) and jeopardize the overall effectiveness of the proposed approach. Therefore, only a finite $c$ value can be practically implemented. A conservative way to predicting a workable range of $c$ is by setting $\exp \left(-c \cdot \lambda / r^{4}\right)=0.5$ where $\lambda \in[0.5,1.5]$ and obtaining $c \in[0.4,1.4] \cdot r^{4}$. It ensures that the indicator functions contain finite values over at least one element for any inflection point (or region). Numerical examples in Section 3 will show that by setting $c=r^{4}$ is effective in capturing the inflection region during the optimization process and obtaining the desirable minimum length scale on the final design.

The proposed indicator functions are computationally cheap. They can be obtained based on the value of the physical field $\bar{\rho}$, the filtered field $\tilde{\rho}$ and its gradient $\nabla \tilde{\rho}$, which are directly available during the optimization process. Moreover, they are differentiable w.r.t. the design variable $\rho$. So are the constraints defined in Section 2.3.

\subsection{Geometric constraints}

Based on the proposed indicator functions in Eqs. (8-9), two geometric constraints are proposed as follows to meet the two requirements in Eqs. (1) and (2) respectively:

$$
\begin{aligned}
& g^{s}=\frac{1}{n} \sum_{i \in \mathbb{N}} I_{i}^{s} \cdot\left[\min \left\{\left(\tilde{\rho}_{i}-\eta_{e}\right), 0\right\}\right]^{2}=0, \\
& g^{v}=\frac{1}{n} \sum_{i \in \mathbb{N}} I_{i}^{v} \cdot\left[\min \left\{\left(\eta_{d}-\tilde{\rho}_{i}\right), 0\right\}\right]^{2}=0,
\end{aligned}
$$

where $n$ is the total number of elements in the discretization set $\mathbb{N}$. By satisfying these two constraints, the value of the filtered field will be larger than the threshold $\eta_{e}$ at the inflection region $\Omega_{1}$ and smaller than the threshold $\eta_{d}$ at $\Omega_{2}$. Therefore, the sufficient condition proposed in Section 1 is satisfied and minimal length scale is expected over the blueprint design.

\footnotetext{
${ }^{3}$ An analytical derivation of the numerical resolution of $\nabla \tilde{\rho}$ is given in Appendix.
} 

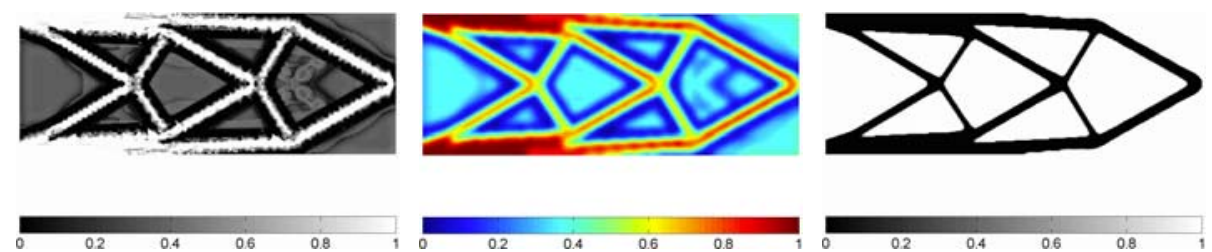

(a) $\rho$

(b) $\tilde{\rho}(r=10)$

(c) $\bar{\rho}(\beta=64, \eta=0.5)$
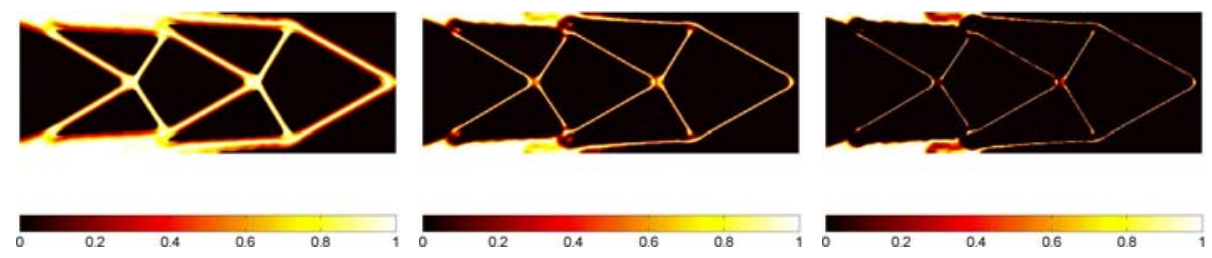

(d) $I^{s}, c=200$

(e) $I^{s}, c=2000$
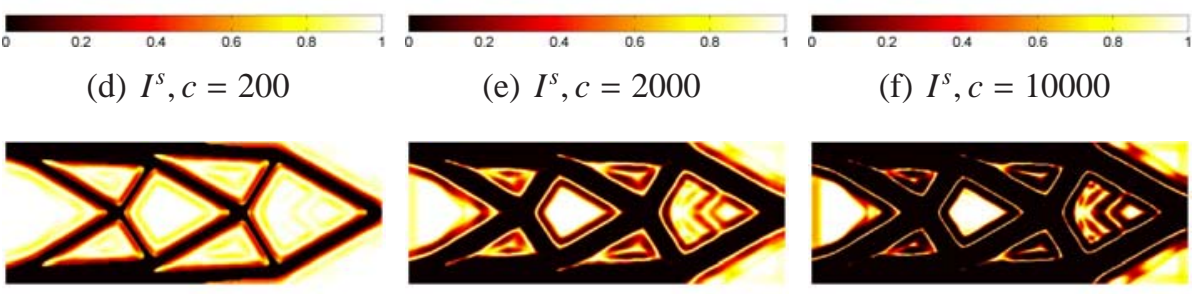

(f) $I^{S}, c=10000$

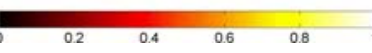

(g) $I^{v}, c=200$

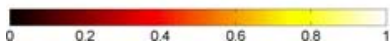

(h) $I^{v}, c=2000$

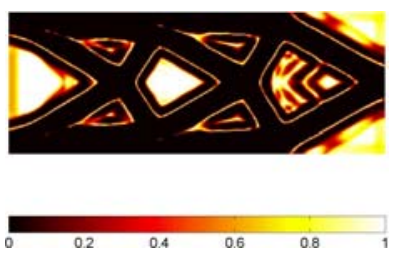

(i) $I^{v}, c=10000$

Figure 2: Structural indictors of an optimized cantilever beam: (a) the optimized design field $\rho$; (b) the filtered field $\tilde{\rho}$; (c) the physical field $\bar{\rho}$; (d-f) the indicators for the solid phase obtained using different $c$ values; (g-f) the indicators for the void. 
In practice, however, the equality constraints Eqs. (10-11) cannot be strictly satisfied due to numerical errors even if the expected minimum length scale has been achieved. The explanation is that by implementing a finite $c$ value, the indicator functions contain (very small) non-zero values $I^{s}, I^{v} \neq 0$ over a "wider" non-inflection regions due to the exponential decay (as the discussed in Section 2.2), where the value of constraints $g^{s}, g^{v}$ are also non-zero. Hence, it is pertinent to relax the constraints as follows:

$$
g^{s} \leq \epsilon \quad \text { and } \quad g^{v} \leq \epsilon
$$

where $\epsilon$ is a small number to compensate the numerical errors. Its appropriate value is discussed later in Section 3.1.5. By adding the relaxed geometric constraints into the standard topology optimization problem Eq. (3), the new formulation becomes:

$$
\begin{array}{rc}
\min : & F(\mathbf{u}(\boldsymbol{\rho}), \boldsymbol{\rho}), \\
\text { s.t. : } & g_{j} \leq 0, \quad j=1: m, \\
: & g^{s} \leq \epsilon, \\
: & g^{v} \leq \epsilon, \\
: & 0 \leq \rho \leq 1 .
\end{array}
$$

Both $g^{s}$ and $g^{v}$ are differentiable w.r.t. the design variable $\rho$ and the optimization problem can be solved using the method of moving asymptotes (MMA) [25] if the first-order sensitivity of the original problem Eq. (3) is available.

The minimum length scale on the final design is determined by the radius of the filter $R$ in Eq. (6), the considered threshold range $\left(\eta_{d}, \eta_{e}\right)$ and the threshold $\eta_{i}$ for the blueprint design. Readers are referred to $[12,24]$ for the details of predicting the minimum length scale based on a linear hat filter for the smoothing operation.

\section{Numerical Examples}

In this section, the proposed geometric constraints are studied using several benchmark topology optimization examples. During the design process, the additional geometric constraints are introduced after an initial topology has been obtained. Such treatment seems to be a necessity for the minimum length-scale type control as introduced here and in refs. $[17,18,19,20]$. The parameter $c$ for the indicator functions is chosen as $c=r^{4}$ for all the examples. More implementation details are provided hereafter. 


\subsection{Compliant mechanism}

\subsubsection{Problem formulation}

The first example is to design a linear elastic compliant gripper [26]. The optimization problem is defined as follows:

$$
\begin{array}{rc}
\text { min. : } & F(\boldsymbol{\rho})=\mathbf{l}^{\top} \mathbf{u}, \\
\text { s.t. : } & \mathbf{K u}=\mathbf{f}, \\
: & \overline{\boldsymbol{\rho}}^{\top} \mathbf{v} \leq V^{*}, \\
: & g^{s} \leq \epsilon, \\
: & g^{v} \leq \epsilon, \\
: & 0 \leq \boldsymbol{\rho} \leq 1,
\end{array}
$$

where $\mathbf{u}$ denotes the displacement vector using finite element discretization, $\mathbf{I}$ contains value 1 at the output degree of freedom and 0 otherwise, $\mathbf{v}$ assembles the volume of all elements, $V_{0}$ and $V^{*}$ are the volume of design domain and the upper allowable volume fraction, respectively. In this example, $E_{\min }=10^{-9} E_{0}$ is used for the SIMP interpolation in Eq. 5.

Fig. 3 shows the design domain $\Omega$ (blue color) and the boundary conditions. On the left side of $\Omega$, the upper and lower corners are clamped. The input force at the center is modeled as a strain-based actuator consisting of a blocking force $F_{i n}$ and an actuator of stiffness $k_{i n}$. On the right side, two symmetric output point at the tip of of the jaw (solid black region) are considered, where two springs of stiffness $k_{\text {out }}$ are attached to simulate the reaction with the target object. The design objective is to maximize the displacement at the tips of the linear elastic gripper under one volume, two geometric and one set of box constraints. The parameters in this example are set as follows: $f_{\text {in }}=1, k_{\text {in }}=0.2, k_{\text {out }}=0.005$, $V^{*}=0.2, E_{0}=1$ and $p=3$.

\subsubsection{Design results}

Fig. 4 compares three optimized mechanism designs with different minimum length scale. The domain is discretized using a uniform quad mesh of resolution

$300 \times 300$. A filter of radius $r=10$ elements (physical length $\frac{1}{30} L$ ) is implemented for all the cases. Fig. 4(a) shows a result without length-scale control, which contains tiny hinges between structural members. Figs. 4(b-c) are two designs obtained by solving the proposed constrained formulation Eq. (13) with considered threshold ranges $\eta \in(0.4,0.6)$ and $(0.3,0.7)$, respectively. The minimum length scale of the two structures increases as the considered threshold range expands. 


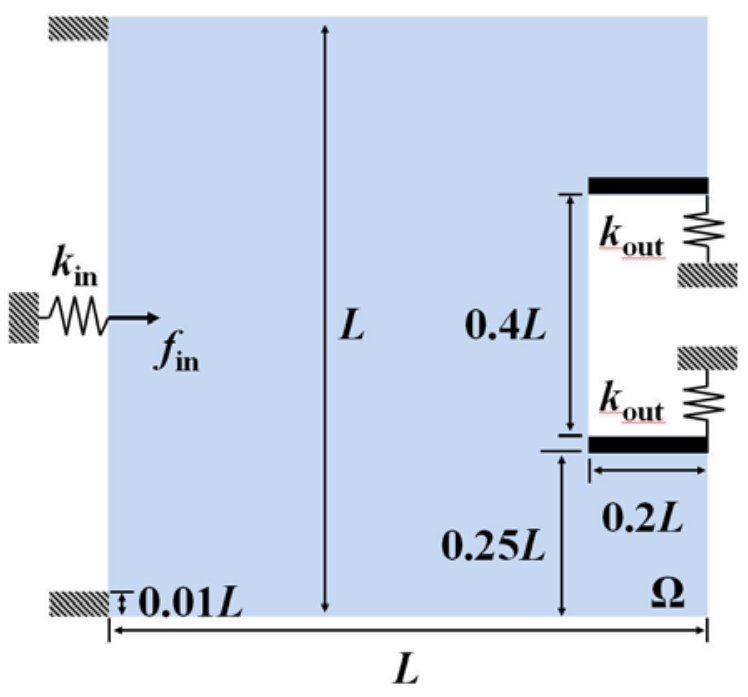

Figure 3: Compliant mechanism design problem: design domain $\Omega$ (blue) and boundary condition.

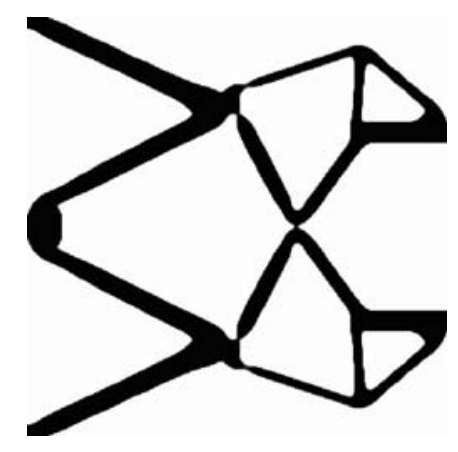

(a) $F=-3.86$

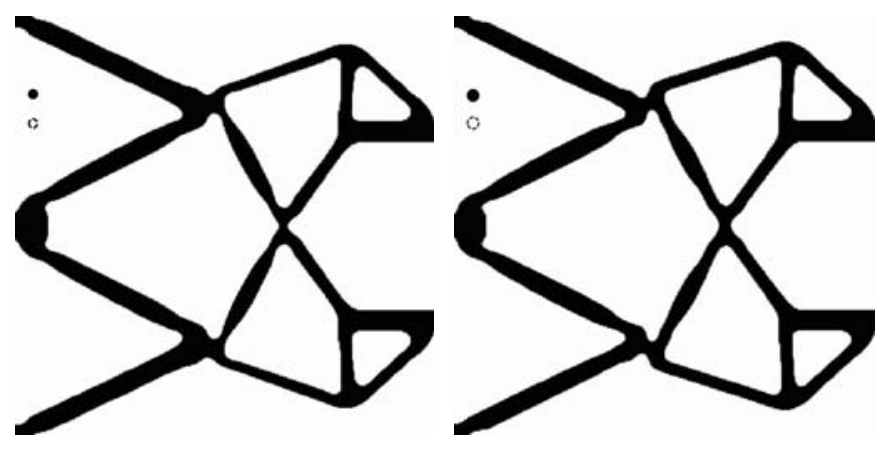

(b) $F=-3.73$

(c) $F=-3.62$

Figure 4: Optimized compliant mechanisms with different minimum length scale: (a) without length-scale control, containing tiny hinges at structural joints; (b) with length-scale control, considered threshold range $\eta \in(0.4,0.6)$; (c) $\eta \in(0.3,0.7)$. 


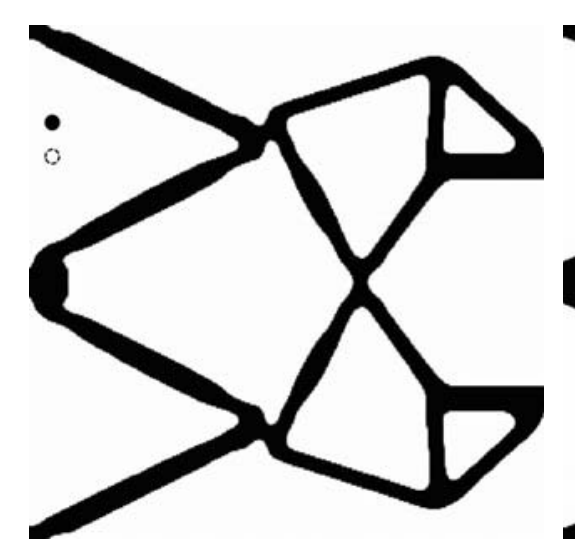

(a) $F=-3.62$

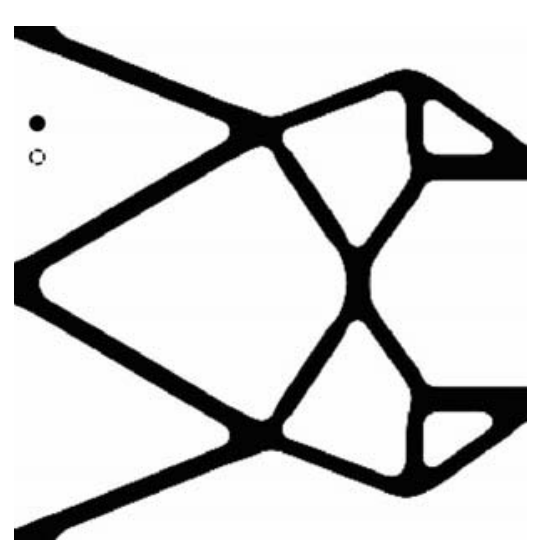

(b) $F=-3.38$

Figure 5: Two optimized designs with same minimum length scale by using: (a) the proposed geometrically constrained formulation, same result as Fig. 4(c); (b) a standard robust formulation [12].

No tiny-hinges appear in the final optimized designs. The exact length scale for material and void phases are indicated by the size of solid and open circles in the figures. These three mechanisms achieve displacement of $F=-3.86,-3.73$ and -3.62 at the output points, respectively. The performance of the compliant gripper degrades as the minimum length scale increases.

In the implementation, $\beta=64$ and the geometric constraints $g^{s}$ and $g^{v} \leq 10^{-8}$ are applied after 75 iterations of the standard topology optimization once a preliminary topology of a compliant gripper is formed. However, it is observed that starting with a grey initial guess and meanwhile imposing the geometric constraints from the beginning can result in a local minima very quickly. In such a case, it is difficult to generate a meaningful topology at the end.

\subsubsection{Performance evaluation w.r.t. geometric variation}

To demonstrate the structural performance w.r.t. geometric variation, the filtered field of the design in Fig. 4(c) is thresholded using different $\eta \in(0.3,0.7)$ and the performance of each realization is plotted as the solid curve in Fig. 6. Functional mechanisms of displacement $D<0$ are observed throughout the considered range $\eta \in(0.3,0.7)$.

This result is compared with another design shown in Fig. 5(b), which is obtained by a standard robust formulation [12]. The latter considers a dilated $\left(\eta_{d}=0.3\right)$, an intermediate (blueprint) $\left(\eta_{i}=0.5\right)$ and an eroded design $\left(\eta_{e}=0.7\right)$ in the problem formulation. The same filtering as in Fig. 4(c) is implemented. 


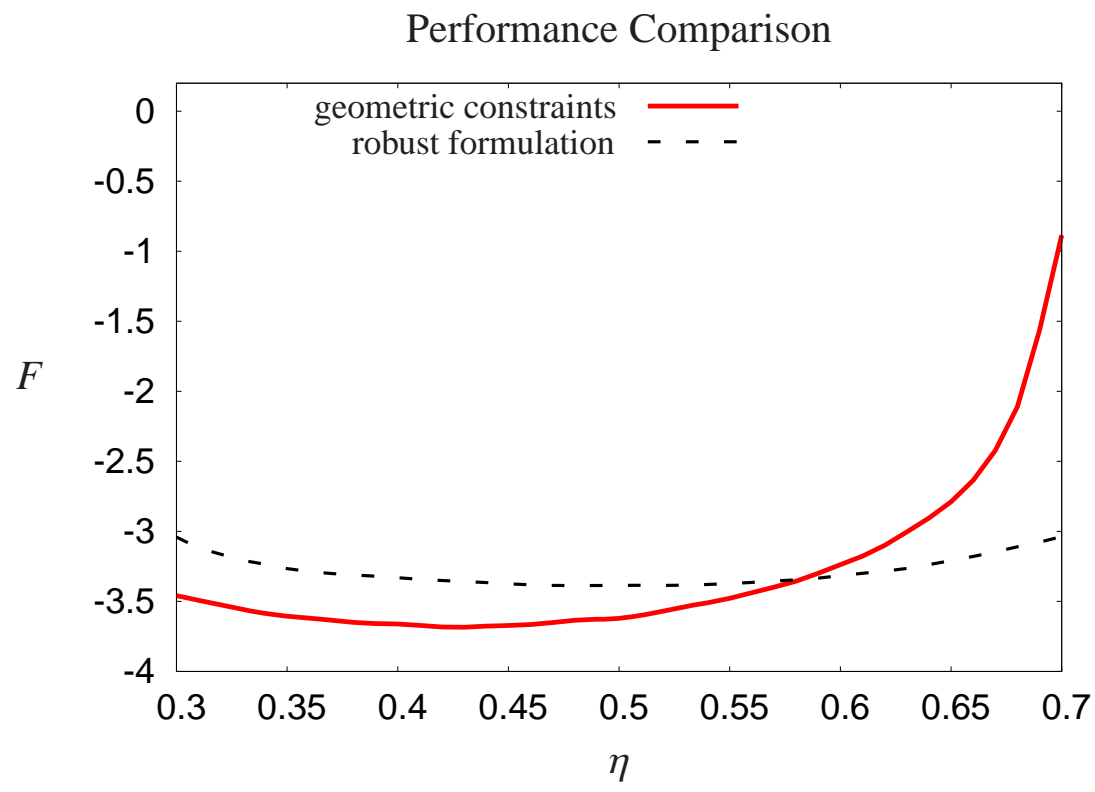

Figure 6: Performance of the two mechanisms shown in Fig. 5 as function of threshold value by using the proposed geometrically constrained formulation (red solid line) and the robust formulation (black dashed line). The considered threshold range for both designs are $\eta \in(0.3,0.7)$.

As shown in Fig. 5, both designs exhibit the same minimum length scale but different structural shape, especially at the joints between structural members. Interestingly, such difference distinguishes the level of performance robustness of a compliant mechanism w.r.t. geometric variations. The performance of the robust design is plotted as the dashed line in Fig. 6. As shown in the figure, the geometrically constrained design outperforms the robust one near the considered design point $\eta=0.5$. However, the robust formulation yield designs which behave less sensitively w.r.t. the member-width variation throughout the overall threshold range.

It is worth to note that the proposed constrained optimization only take the performance of the blueprint (intermediate) design into account and imposing length scale by the proposed geometric constraints does not necessarily guarantee a robust performance w.r.t. geometric variation. One can obtain designs of robust performance by using a robust formulation $[11,12,13]$. However, minimum length scale may not be realized on the optimized blueprint as the other realizations in the considered threshold range exhibit different topologies [12]. Regarding computational efficiency, only one finite element analysis per design iteration is required 


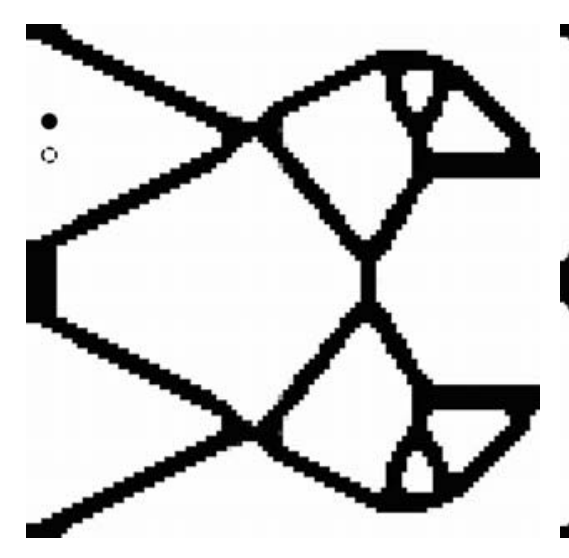

(a) $100 \times 100$ mesh

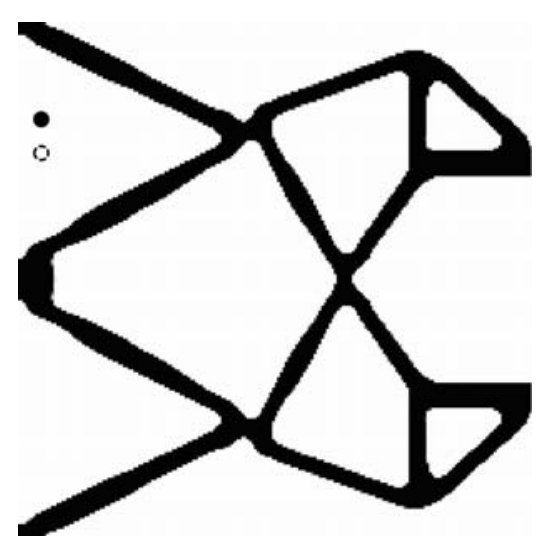

(b) $200 \times 200$ mesh

Figure 7: Designs with different discretization: (a) $100 \times 100$ mesh, $r=3.5$ elements; (b) $200 \times 200$ mesh, $r=7$ elements. The considered threshold range is $(0.3,0.7)$.

by using the proposed formulation, while several analysis ${ }^{4}$ are needed when the robust formulation is adopted.

\subsubsection{Design with different discretization}

The proposed scheme can be applied to design structures of different resolutions. First, a coarse mesh of $100 \times 100$ elements is used and the optimized mechanism is shown in Fig. 7(a). In this example, constants $r=3.5$ elements, $c=150$ and $\beta=64$ are implemented. The considered threshold range is still $\eta \in(0.3,0.7)$. Comparing to the previous design as shown in Fig. 5(a) (obtained with $r=10$ elements and $c=10000$ ), the current optimization process utilizes smaller $r$ and $c$ values, which lead to a larger numerical error for the indictors in capturing the inflection region. As a result, the geometric constraints is further relaxed to $\epsilon=10^{-6}$ in order to be satisfied. It is found that by increasing $c$ in the range $c \in[150,600]$ and meanwhile slightly tightening the geometric constraints in the range $\epsilon \in\left[10^{-6}, 10^{-7}\right]$ can yield designs with the same minimum length scale. However, if $c \geq 700$ is chosen, the value of $I^{s}$ and $I^{v}$ are too small $\left(I^{s}, I^{v} \approx 0\right)$ at some inflection regions and it is very difficult to obtain desirable outcome in such a case.

Fig. 7(b) shows another optimized design of the same minimum length scale

\footnotetext{
${ }^{4}$ The number of finite element analysis by using a robust formulation is equal to the number of design realizations considered in the problem formulation.
} 
but with a finer resolution $200 \times 200$. The parameters used in optimization are $r=7$ elements, $c=2400, \eta \in(0.3,0.7)$ and $\epsilon=10^{-7}$. In this example, as the mesh resolution becomes finer and the filter covers more elements, it is found the workable $c$ value spans a larger range between $c \in[1500,3500]$ for satisfied geometric constraints $g^{s}, g^{v} \leq 10^{-7}$ and the same minimum length scale.

\subsubsection{Discussion on choosing $c$ and $\epsilon$}

In practice, the parameter $c$ is chosen based on the numerical accuracy in representing the gradient of a filtered field. The indicator functions should have finite values (e.g. $I^{S}, I^{v} \geq 0.1$ ) to capture the discretized inflection regions (represented by at least one element) during the optimization process. Otherwise, it will be difficult to achieve the desirable length scale if the values of $I^{s}, I^{v}$ at the inflection regions are too small (e.g. $I^{s}, I^{v}<10^{-2}$ ). It is advised to apply a large $c$ value if the numerical error is small, and vice versa. Based on the analytic derivation of the numerical resolution in Appendix, a conservative setting $c=r^{4}$ is found effective for all the numerical examples in this paper.

Due to numerical errors, the geometric constraints are relaxed in order to be satisfied. Generally, a small filter radius $r$ (elements) accompanied with a small $c$ causes a large numerical error in representing the inflection regions by the indicator function. Hence, a further relaxation on the geometric constraints is required, and vice versa. Fig. 8 records a set of $\epsilon$ in the mechanism design problem with a $200 \times 200$ mesh, for which both satisfied constraints and minimum length scale are obtained for different $r$ and $c\left(c=r^{4}\right)$. It is found that similar results can be obtained by slightly varying $\epsilon$ around the curve. But over-relaxing the constraints will fail in imposing the desirable length scale on the final designs. Numerical investigation shows that this curve can serve as a reference to choose $\epsilon$ for different $r$, provided that $c=r^{4}$ is used. All the other examples in this paper are implemented according to it and satisfactory results are obtained. However, further research is needed to eliminate the parameter tuning in the proposed method. 


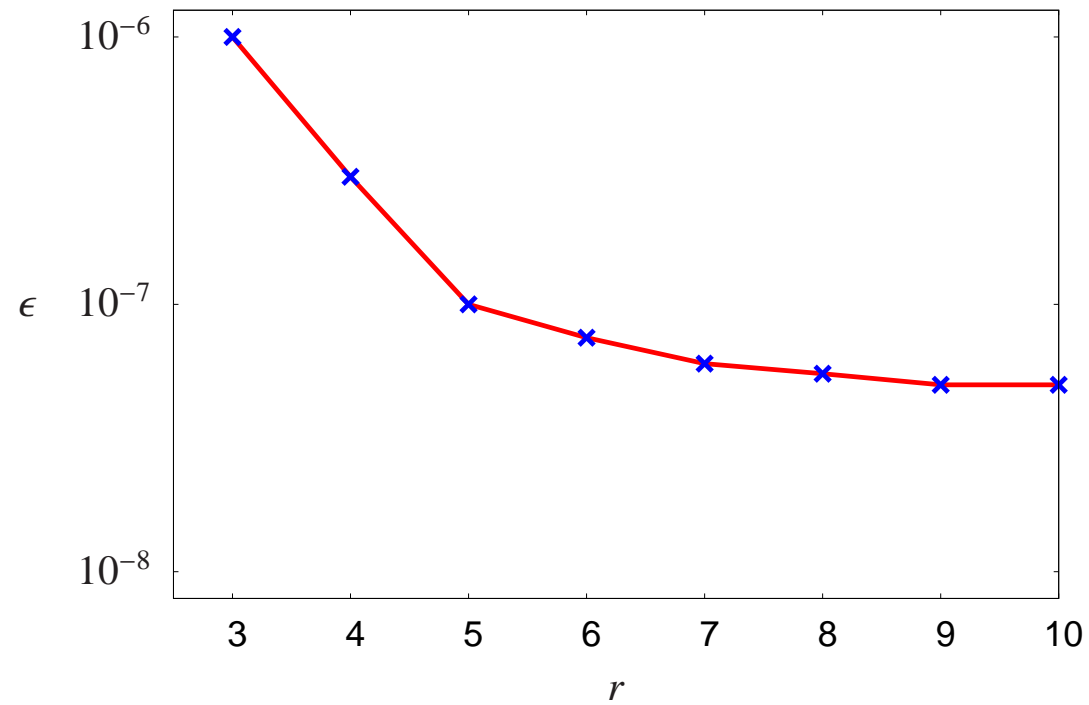

Figure 8: Choosing $\epsilon$ for different $r$ and $c=r^{4}$. Each marker is recorded when both minimum length scale and satisfied geometric constraints are achieved in the mechanism design example with mesh $200 \times 200$.

\subsection{Heat conduction}

\subsubsection{Problem formulation}

The second example studies a heat conduction problem. The discretized formulation is given as follows:

$$
\begin{array}{rc}
\operatorname{min.}: & F(\boldsymbol{\rho})=\mathbf{p}^{\top} \mathbf{t}, \\
\text { s.t. : } & \mathbf{C t}=\mathbf{p}, \\
: & \overline{\boldsymbol{\rho}}^{\top} \mathbf{v} \leq V^{*}, \\
: & g^{s} \leq \epsilon, \\
: & g^{v} \leq \epsilon, \\
: & 0 \leq \boldsymbol{\rho} \leq 1,
\end{array}
$$

where $\mathbf{t}$ denotes the temperature vector, $\mathbf{p}$ corresponds to uniform heating over the domain with value 1 at all degrees of freedom and $\mathbf{C}$ is the conductivity matrix. The SIMP model in Eq. (5) with $p=3$ is used for computing the conductivity matrix by replacing the stiffness $\mathbf{K}$ with conductivity $\mathbf{C}$. The conductivity is set to $E_{0}=1$ and $E_{\min }=10^{-3}$ for the solid and void respectively. 


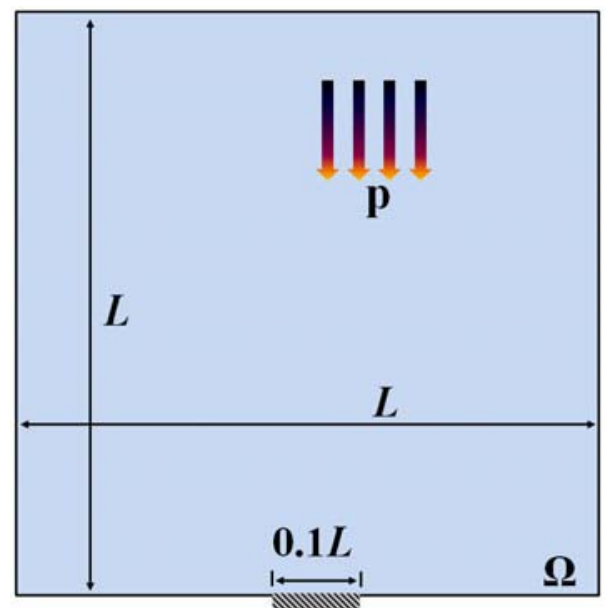

Figure 9: Heat conduction problem: design domain $\Omega$ (blue) and boundary condition.

Fig. 9 shows the design domain $\Omega$ (blue color) and the boundary conditions. The domain is isolated at the boundary except that a heat sink with temperature 0 is attached at the central bottom part of the domain. In the implementation, only a half of the design domain is considered due to symmetric boundary conditions. The upper volume fraction is set as $V^{*}=0.3$.

\subsubsection{Design results}

Fig. 10 records several snapshots of an optimization process before and after the geometrical constraints are applied. . In this example, the domain is discretized using a uniform quad mesh of resolution $300 \times 300$. Constants $r=10$ elements, $\epsilon=10^{-8}$ and $\beta=32$ are implemented. The considered threshold range is $\eta \in(0.25,0.75)$.

The design shown in Fig. 10(a) is obtained after 50 iteration by solving a standard topology optimization problem without geometric constraints. The constraints are highly violated when they are first applied due to the intermediate value (grey) elements in Fig. 10(a). However, the constraints tend to suppress the grey regions and the filtered field is gradually regularized as shown in Fig. 10(b-f). Topological change is observed during the constrained optimization process. The final optimized design is shown in Fig. 11(a), which achieves an objective value of $F=2.86$.

As a comparison, another design result with a smaller length scale is shown in Fig. 11(b). It exhibits more structural details with a smaller length scale and achieves a better objective value of $F=2.37$. For this example, a smaller filter 


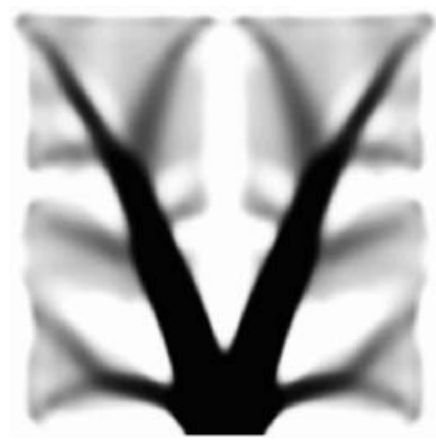

(a)

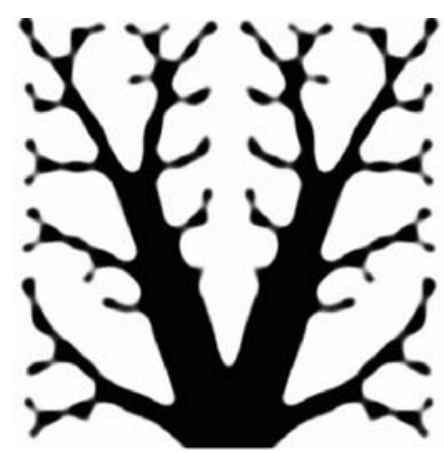

(d)

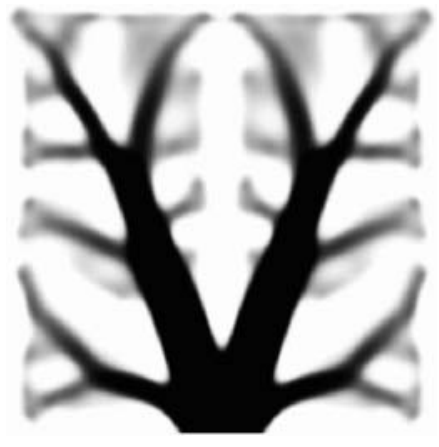

(b)

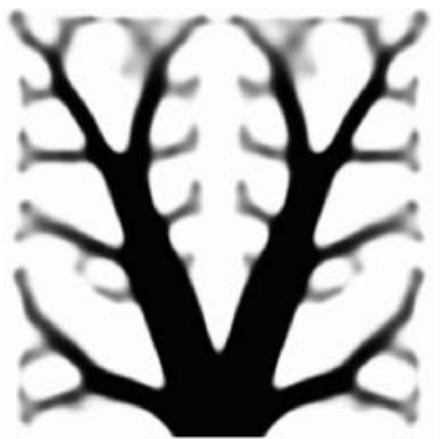

(c)

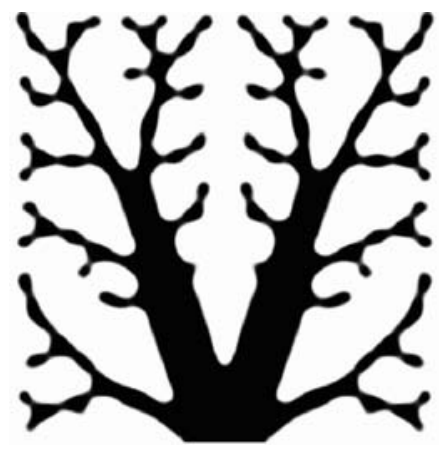

(e)

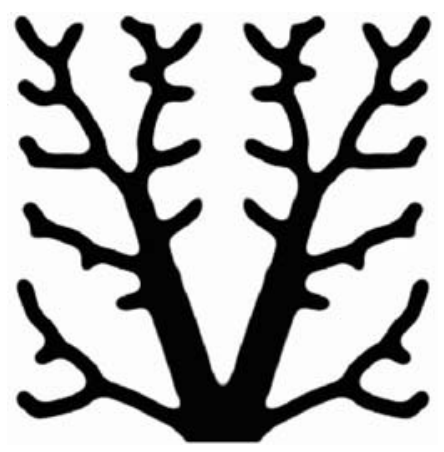

(f)

Figure 10: Optimization process of the heat conduction problem with geometric constraints, threshold range $\eta \in(0.25,0.75)$ : (a) the intermediate design before applying the geometric constraints; (b-f) design evolution with constraints applied.

radius $r=5$ elements is chosen and the considered threshold range is still $\eta \in$ $(0.25,0.75)$. The geometric constraints $g_{s}, g_{v} \leq 10^{-7}$ are satisfied when achieving the expected length scale.

\subsubsection{Discussion}

Due to the additional geometrical constraints, the design space of the proposed formulation becomes more restrictive than that in the standard formulation. The final optimized result tends to be initial-design dependent although topological change is observed during the design process. In order to fully leverage the capability of topology optimization and meanwhile to support the minimum lengthscale control, it is desirable to apply the geometric constraints later in the optimization process after an initial topology has formed.

For the heat conduction problem with distributed thermal load, the standard 


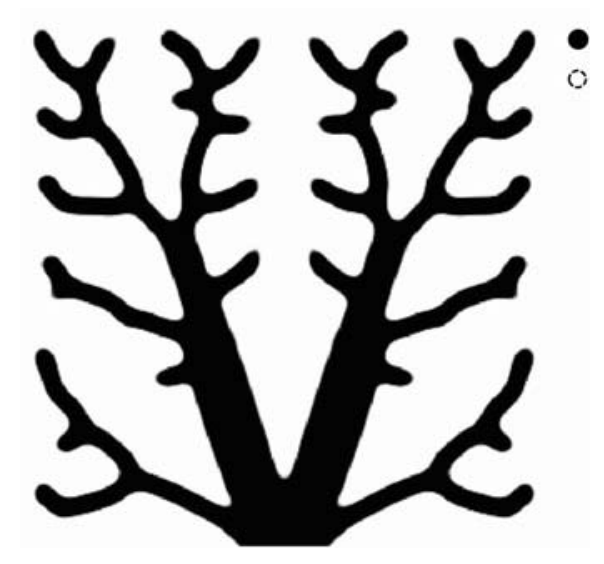

(a) $r=10, F=2.86$

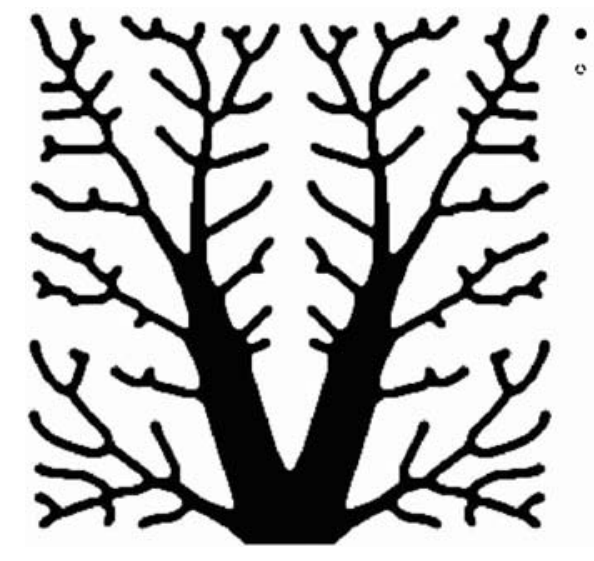

(b) $r=5, F=2.37$

Figure 11: Optimized blueprint designs of the heat conduction problem with different length scale: (a) $r=10, H=2.86$; (b) $r=5, H=2.37$; the considered threshold range is $(0.25,0.75)$ for both designs.

topology optimization formulation [2] usually results in an optimized design containing elements of intermediate values. Because of these grey elements, the geometrically constrained problem starts with a highly infeasible design and the optimization process may end up with a physically unexpected (local minima) solution, e.g. the final design containing connected structural members. Properly normalizing and balancing the sensitivities of the objective functional and the constrains can avoid such issue and obtain meaningful results.

\subsection{Slow light waveguide}

The problem of designing a dispersion engineered slow light waveguide is investigated in this section by applying the proposed constraints. Fig. 12 shows the design domain $\Omega$, which is discretized using a $512 \times 32$ quad mesh. A slow light waveguide is obtained by minimizing the errors between actual group index $n_{g}$ and a prescribed group index $n_{g}^{*}$ in a given wavenumber range $k$. For conciseness, the original problem formulation is not repeated in this paper. Readers are

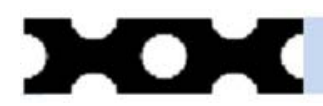

$\Omega$

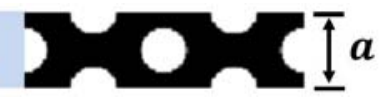

Figure 12: Slow light waveguide problem: a single design unit in a (vertically) periodic structure, design domain $\Omega$. 
referred to $[27,28]$ for more details.

It has been demonstrated that robust topology optimization formulations cannot always guarantee the same topology for all the considered design realizations in the problem formulation w.r.t. geometric variations, although equally good performances can be obtained [27]. Fig. 13 shows such a result by solving a worst-case based robust formulation [12] considering three physical design realizations thresholded by $\eta=0.35,0.5$ and 0.65 . In this example, the filter radius $r=3.75$ elements, $\epsilon=10^{-6}$ and $\beta=50$ are implemented. As shown in Fig. 13(c), the dilated $(\eta=0.35)$, eroded $(\eta=0.65)$ and blueprint $(\eta=0.5)$ designs are equally optimized with the expected group index. However, they exhibit different topologies as the contour plot shown in 13(b), where some small holes shown in 13(a) vanish in the eroded design. No strict length scale can be identified on the blueprint design.

Using the result in Fig. 13(a) as the initial guess for the proposed scheme, another optimized slow light waveguide is obtained as shown in Fig. 14(a). This new result possesses minimum length scale in both solid and void phases as the contours of the eroded, blueprint and dilated designs in Fig. 14(b) demonstrate a same topology. The corresponding performance is compared in Fig. 14(c). The blueprint $(\eta=0.5)$ demonstrates a good performance regarding the desirable group index $n_{g}^{*}=25$ in the design wavenumber range of $k \in[0.3875,0.4625]$. $2 \pi / a$. However, the performance of the other realizations are either degraded or destroyed due to geometric variations. The physical realizations threshold at $\eta=0.45$ and $\eta=0.55$ correspond to approximately $15 \mathrm{~nm}$ manufacturing error in practice. This example demonstrates that imposing minimum length scale does not result in a functional slow light waveguide throughout the considered threshold range. The design performance is highly sensitive w.r.t geometric variation.

Combining the worst-case based robust formulation and the proposed geometric constraints result in a design shown in Fig. 15. Equally optimized performance is achieved for the considered three designs and minimum length scale is identified on the blueprint design. However, because the robust formulation here only takes three designs into account, the other intermediate realizations (e.g. $\eta=0.45,0.55$ ) still do not behave as well as the blueprint. This issue may be alleviated by including more realizations in the formulation.

\section{Conclusions}

A topology optimization approach with geometric constraints is presented to design structures that possess strict minimum length scale. The constraints are 
formulated based on structural indicator functions, which are defined on the regularized filtered and physical fields in a three-field topology optimization scheme. They are computationally cheap and differentiable w.r.t. the design variable. The constrained optimization problem is solved using mathematical programming. No additional finite element analysis is required.

In order to utilize this approach effectively, it is advised to provide a good initial guess for the constrained optimization. One pertinent way is by adding the constraints later into the standard topology optimization process after an initial topology has formed. It is found difficult to obtain efficient designs if the initial guess for the constrained optimization is far from an admissible feasible design. One limitation of the proposed method is that parameters $c$ and $\epsilon$ must be chosen properly based on the level of numerical accuracy in representing the underlying structure. However, strategies based on numerical investigation are suggested to set those parameters. It is targeted as future work to formulate a scheme without parameter tuning.

The structural indicator functions proposed in this paper can be replaced by other representations, such as a medial-axis (or media-zone) based scheme. A differentiable representation is necessary to carry out a full sensitivity analysis and to solve the optimization problem in a meaningful way.

Numerical examples in this paper reveal the fact that imposing minimum length scale does not necessarily guarantee robust performance w.r.t. geometric variation. For the mechanics problem, workable compliant mechanisms are obtained w.r.t. structural membrane-width change. But for the dispersion engineered slow light waveguide problem, for which the physical performance is very sensitive to geometric variations, a degraded performance is observed for eroded and dilated structures even if they share the same topology to a well-optimized blueprint design. Combining a worst-case based robust formulation and the proposed geometric constraints results in designs with equally good performance for the considered design realizations besides the desirable minimum length scale. Future work will investigate how to efficiently achieve a robust performance w.r.t. a range of geometric variations and the minimum length scale at the same time in topology optimization.

\section{Acknowledgement}

The authors acknowledge the financial support received from the Hans Christian Ørsted Postdoctoral grant at the Technical University of Denmark, from the NextTop project sponsored by the Villum foundation, from the "LaScISO" project 
(Grant No. 285782) and from the ERC Starting Grant (INNODYN). The authors thank Joe Alexandersen at DTU TopOpt group for discussion and re-implementing the standard heat conduction problem which is originally given in the book [2].

\section{Appendix: Numerical resolution in representing $\nabla \tilde{\rho}$ in a uniform finite ele- ment mesh}

In a discretized filtering-threshold topology optimization scheme, $\nabla \tilde{\rho}=0$ does not hold precisely over the entire inflection region of a filtered field because of numerical errors. In this section, the numerical resolution and the error of representing $\nabla \tilde{\rho}$ is discussed.

With a uniform discretization with unit length $(h=1)$, the linear-hat filtering can be described by a convolution function $w=(1-|x| / r) / r$ over the domain $[-r, r]$ covered by the filter, where $r$ is the filter radius [24]. For a feature of width

$l<r$ as shown in Fig. 16, the expression of the filtered field and its gradient at $x \in\left[x_{0}, x_{0}+l\right]$ are computed as follows:

$$
\tilde{\rho}(x)=\int_{x_{0}}^{x} \frac{1}{r}\left(1-\frac{x-x_{0}}{r}\right) d x+\int_{x}^{x_{0}+l} \frac{1}{r}\left(1-\frac{x_{0}+l-x}{r}\right) d x,
$$

and hence

$$
\nabla \tilde{\rho}(x)=\frac{2 x_{0}+l}{r^{2}}-\frac{2 x}{r^{2}}, \quad x \in\left[x_{0}, x_{0}+l\right] .
$$

Assuming that the field values $\rho$ and $\tilde{\rho}$ are constant inside each element, the numerical resolution of representing $\nabla \tilde{\rho}$ is equal to $1 / r^{2}$ in this $1 \mathrm{D}$ example. When computing $\nabla \tilde{\rho}$ using finite difference, the numerical error of $\nabla \tilde{\rho}$ at the inflection regions is approximately of the same order of magnitude to the above resolution, which dependent on the radius of the filter $r$. Note that $r$ is in the unit of number of elements instead of a physical length. For 2D problems, it is observed that the value of $\nabla \tilde{\rho}$ at the inflections ranges approximately between $\nabla \tilde{\rho} \in[0,1.5] / r^{2}$, for which the upper bound slightly varies among different structural layouts.

\section{Reference}

[1] M. Bendsøe, N. Kikuchi, Generating optimal topologies in structural design using a homogenization method, Computer Methods in Applied Mechanics and Engineering, 71 (1988) 197-224.

[2] M. Bendsøe, O. Sigmund, Topology Optimization - Theory, Methods and Applications, Spinger, (2003). 
[3] O. Sigmund, K. Maute, Topology optimization approaches, Structural and Multidisciplinary Optimization, 48 (2013) 1031-055.

[4] C. BW. Pedersen, P. Allinger, Industrial implementation and applications of topology optimization and future needs, IUTAM Symposium on Topological Design Optimization of Structures, Machines and Materials Solid Mechanics and Its Applications, 137 (2006) 229-238.

[5] M. Zhou, R. Fleury, S. Patten, N. Stannard, D. Mylett, S. Gardner, Topology Optimization - Practical Aspects for Industrial Applications, in Proc. of 9th World Congress on Structural and Multidisciplinary Optimization, (2011).

[6] D. Brackett, I. Ashcroft, R. Hague, Topology optimization for additive manufacturing, 22nd Annual International Solid Freeform Fabrication Symposium, (2011) 348-362.

[7] M. Jansen, B.S. Lazarov, M. Schevenels, O. Sigmund, On the similarities between micro/nano lithography and topology optimization projection methods, Structural and Multidisciplinary Optimization, 48 (2013) 717-730.

[8] M. Zhou, B.S. Lazarov, O. Sigmund, Topology optimization for optical projection lithography with manufacturing uncertainties, Applied Optics, 53 (2014) 2720-2729.

[9] T.A. Poulsen, A new scheme for imposing a minimum length scale in topology optimization, International Journal for Numerical Methods in Engineering, 57 (2003) 741-760.

[10] J.K. Guest, J.H. Prévost,T. Belytschko, Achieving minimum length scale in topology optimization using nodal design variables and projection functions, International Journal for Numerical Methods in Engineering, 61 (2004) 238254.

[11] O. Sigmund, Morphology-based black and white filters for topology optimization, Structural and Multidisciplinary Optimization, 33 (2007) 401-424.

[12] F. Wang, B. Lazarov, O. Sigmund, On projection methods, convergence and robust formulations in topology optimization, Structural and Multidisciplinary Optimization, 43 (2011) 767-784. 
[13] M. Schevenels, B.S. Lazarov, O. Sigmund, Robust topology optimization accounting for spatially varying manufacturing errors, Computer Methods in Applied Mechanics and Engineering, 200 (2011) 3613-3627.

[14] B.S. Lazarov, M. Schevenels, O. Sigmund, Topology optimization with geometric uncertainties by perturbation techniques, International Journal for Numerical Methods in Engineering, 90 (2012) 1321-1336.

[15] M.Y. Wang, X. Wang, D. Guo, A level set method for structural topology optimization, Computer Methods in Applied Mechanics and Engineering, 192 (2003) 227-246.

[16] S. Chen, M.Y. Wang, A. Liu, Shape feature control in structural topology optimization, Computer-Aided Design, 40 (2008) 951-962.

[17] X. Guo, W. Zhang, W. Zhong, Explicit feature control in structural topology optimization via level set method, Computer Methods in Applied Mechanics and Engineering, 272 (2014) 354-378.

[18] W. Zhang, W. Zhong, X. Guo, An explicit length scale control approach in SIMP-based topology optimization, Computer Methods in Applied Mechanics and Engineering, 282 (2014) 71-86.

[19] G. Michailidis, Manufacturing Constraints and Multi-Phase Shape and Topology Optimization via a Level-Set Method, Optimization and Control, Ecole Polytechnique X, (2014), available at: https://tel.archivesouvertes.fr/pastel-00937306.

[20] G. Allaire, F. Jouve, G. Michailidis, Thickness control in structural optimization via a level set method, (2014) < hal $-00985000>$, available at: https://hal.archives-ouvertes.fr/hal-00985000.

[21] M. Zhou, M.Y. Wang, Engineering feature design for level set based structural optimization, Computer-Aided Design, 45 (2013) 1524-1537.

[22] J.K. Guest, Imposing maximum length scale in topology optimization, Structural and Multidisciplinary Optimization, 37 (2009) 463-473.

[23] M. Bendsøe, Optimal shape design as a material distribution problem, Structural Optimization, 1 (1989) 193-202. 
[24] X. Qian, O. Sigmund, Topological design of electromechanical actuators with robustness toward over- and under-etching, Computer Methods in Applied Mechanics and Engineering, 253 (2013) 237-251.

[25] K. Svanberg, The method of moving asymptotes - a new method for structural optimization, International Journal for Numerical Methods in Engineering, 24 (1987) 359-397.

[26] O. Sigmund, On the Design of Compliant Mechanisms Using Topology Optimization, Mechanics of Structures and Machines, 25 (1997) 493-524.

[27] F. Wang, J.S. Jensen, O. Sigmund, Robust topology optimization of photonic crystal waveguides with tailored dispersion properties, Journal of the Optical Society of America B, 28 (2011) 387-397.

[28] F. Wang, J.S. Jensen, O. Sigmund, High-performance slow light photonic crystal waveguides with topology optimized or circular-hole based material layouts, Photonics and Nanostructures-Fundamentals and Applications, 10 (2012) 378-388. 


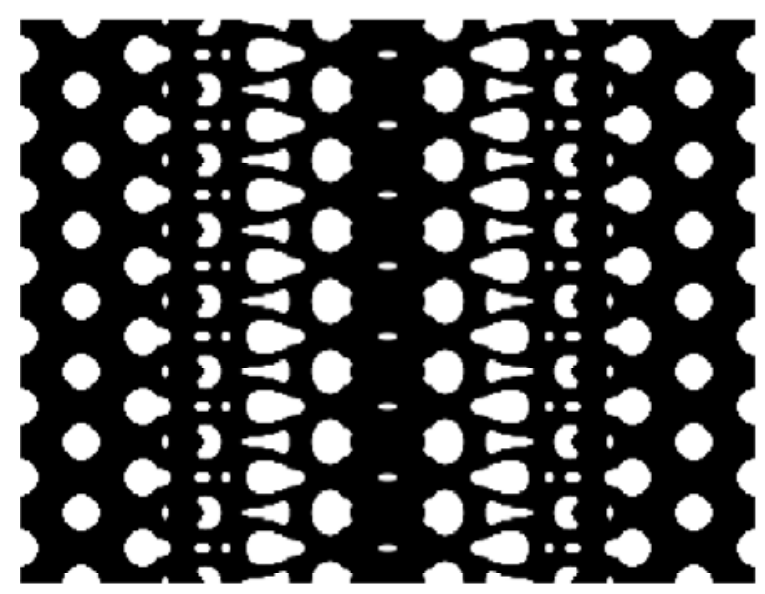

(a)

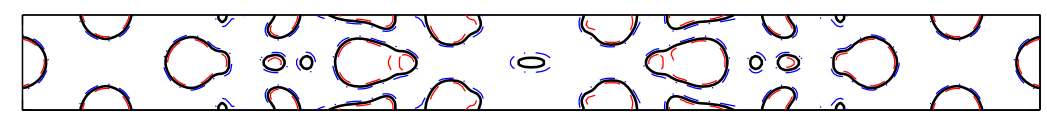

(b)

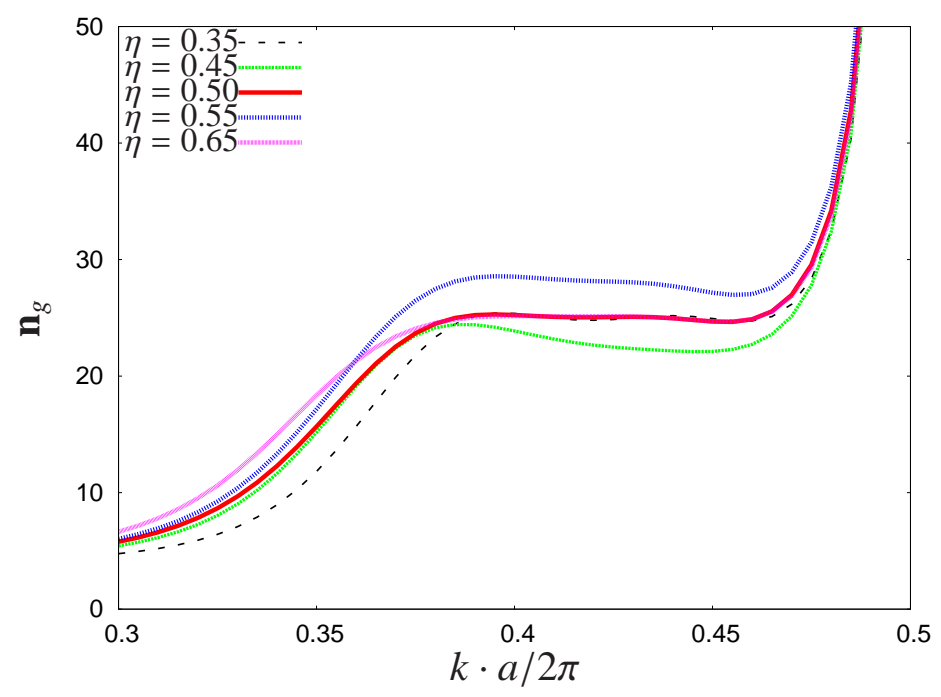

(c)

Figure 13: Optimized slow light waveguide for $n_{g}=25$ in $k \in[0.3875,0.4625] 2 \pi / a$ by using a robust formulation. (a) the waveguide composed of 8 repeated cells; (b) contour plots of the blueprint (in black bold lines), dilated design realization (in blue dashed lines) and eroded design realization (in red dash-dotted lines); (c) performance evaluation by varying structural membranewidth. 


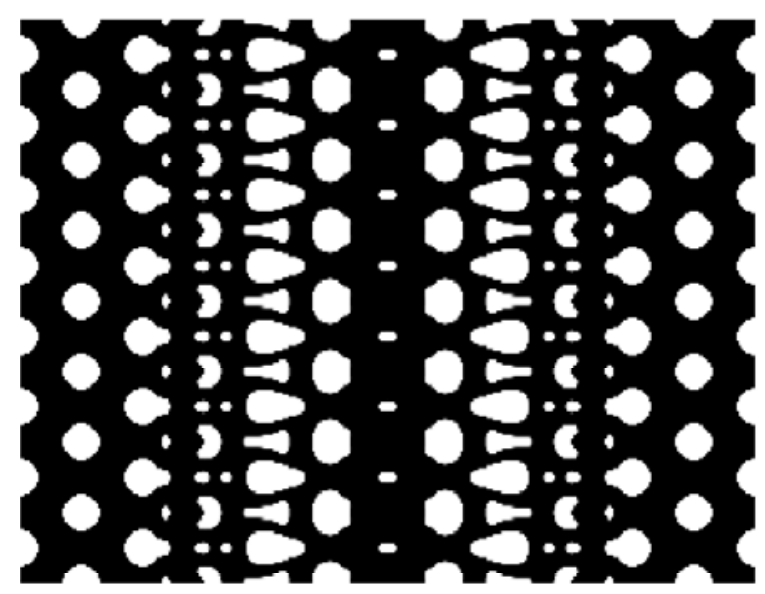

(a)

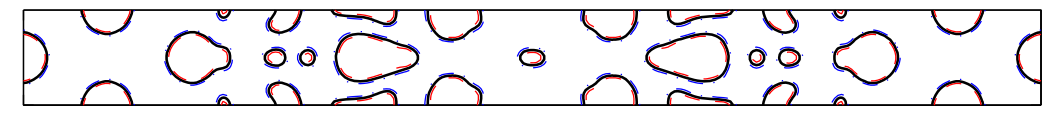

(b)

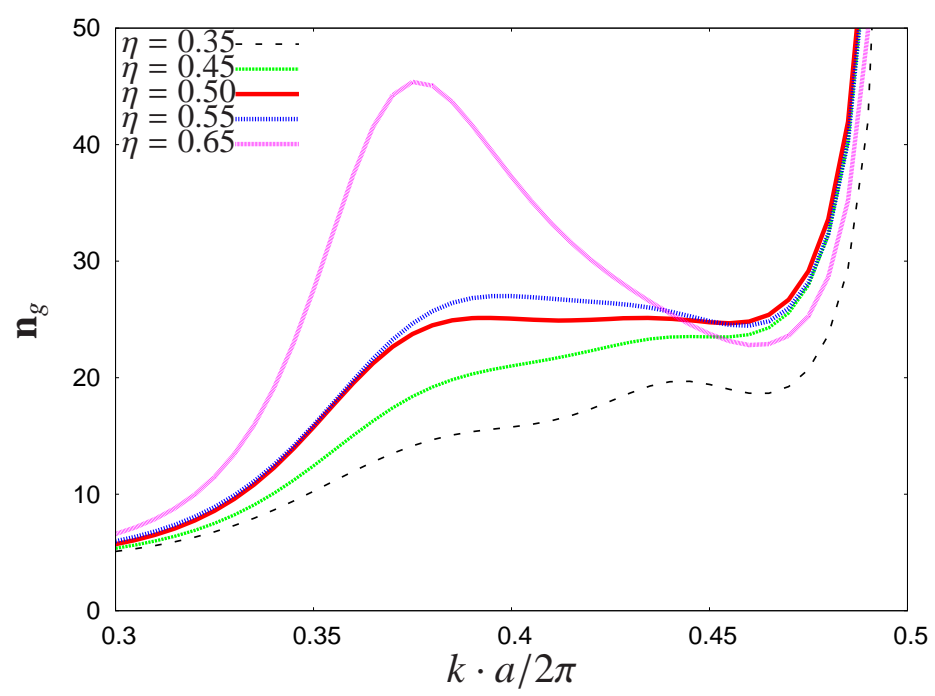

(c)

Figure 14: Optimized slow light waveguide by using the proposed geometric constraints. (a) the waveguide composed of 8 repeated cells; (b) contour plots of the blueprint (in black bold lines), dilated design realization (in blue dashed lines) and eroded design realization (in red dash-dotted lines); (c) performance evaluation by varying structural membrane-width. 


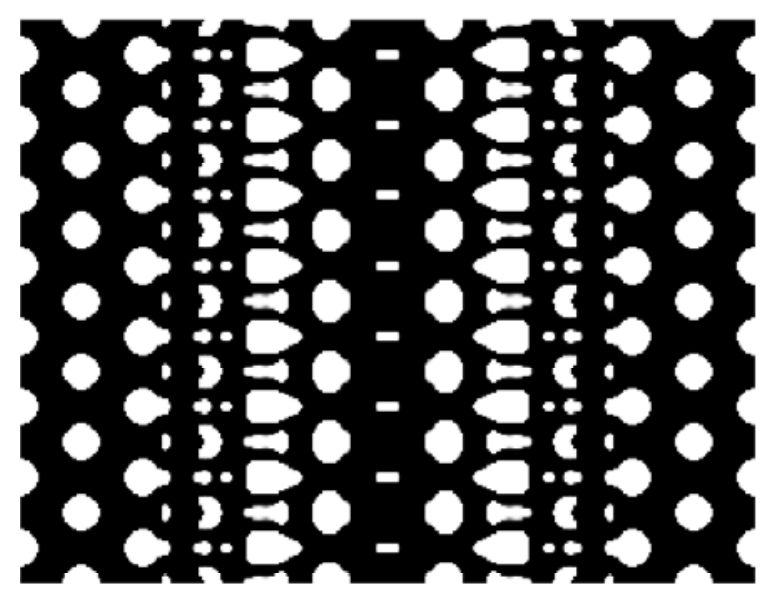

(a)

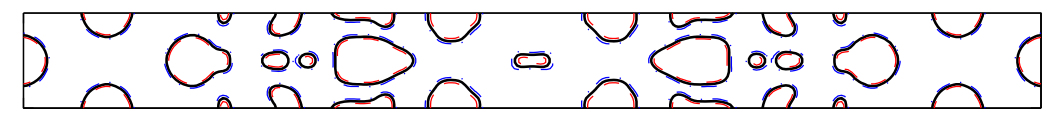

(b)

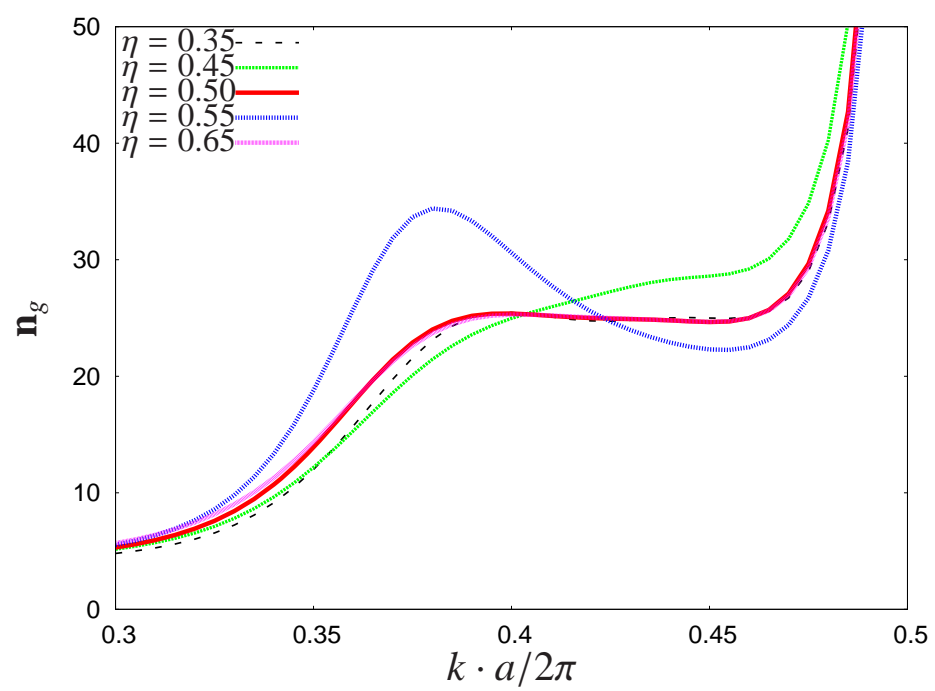

(c)

Figure 15: Optimized slow light waveguide by using both a robust formulation and geometric constraints. (a) the waveguide composed of 8 repeated cells; (b) contour plots of the blueprint (in black bold lines), dilated design realization (in blue dashed lines) and eroded design realization (in red dash-dotted lines); (c) performance evaluation by varying structural membrane-width. 


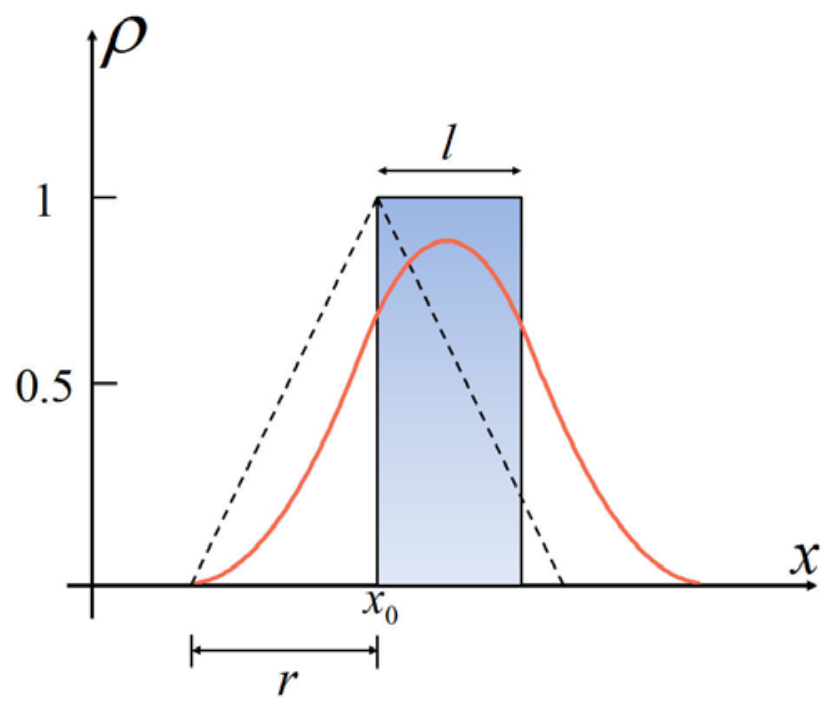

Figure 16: Filtering a feature of width $l<r$ using a linear-hat filter. The red solid curve denotes the filtered field $\tilde{\rho}$. 